\title{
Stepped care for depression and anxiety in visually impaired older adults: multicentre randomised controlled trial
}

Due to an editorial error, the legend for figure 2 in this research paper (BMJ 2015;351:h6127, doi:10.1136/bmj.h6127) is incorrect. The control group is represented by the red dotted line and the intervention group corresponds to the solid blue line in the figure, not the other way round as the legend describes. 